\title{
A THERMODYNAMIC MODEL OF GRAIN BOUNDARY SEGREGATION FOR ATOMISTIC CALCULATIONS
}

\author{
T. NOWICKI ${ }^{(1)}, J_{.}-\mathrm{C}$. JOUD* and M. BISCONDI \\ Ecole Nationale Supérieure des Mines, 158 Cours Fauriel, F-42023 Saint \\ Etienne Cedex 2, France \\ "Laboratoire de Thermodynamique et Physico-Chimie Métallurgiques, UA \\ 29. ENSEEG, BP. 75, F-38402 Saint Martin d'Hères Cedex, France
}

\begin{abstract}
Résumé : Les modèles thermodynamiques classiques ne permettent pas une description réaliste de la ségrégation intergranulaire, car ils ne prennent pas en compte les différences entre les caractéristiques énergétiques et géométriques des différents sites effectifs de ségrégation. Dans cet article, nous proposons un nouveau modèle thermodynamique échappant à la critique précédente et permettant de calculer la concentration intergranulaire en soluté, en fonction de la structure de l'interface et de la température. On donne aussi une méthode de calcul de la tension interfaciale en fonction de l'énergie intergranulaire.
\end{abstract}

Summary : Existent thermodynamic models do not allow a realistic description of segregation. The major reason for this is that classical models of segregation do not take into account the differences between the energetic and geometrical characteristics among the different effective segregation sites. In this paper we propose a new thermodynamical model which suppress mentioned problem and enables a determination of interface solute concentration depending on interface structure and temperature. A method for calculation of grain boundary tension as a function of grain boundary energy is also described.

\section{Introduction}

Development of defect calculation techniques such as molecular statics and molecular dynamics associated to the progress made in construction of interatomic potentials allow the calculations of structure and energy of grain boundaries in multi-component systems. Such calculations are of particular interest in the systems subjected to interfacial segregation.

There are two major difficulties in use of the results of atomistic calculations for segregation prediction in different temperatures :

1) the classical thermodynamic segregation models (such as Mc Lean model) do not take into account the existence of different types of segregation sites ;

2) it is not easy to transform the quantities obtained from atomistic calculations to those used by classical thermodynamic models.

In our paper we propose a new thermodynamic description of grain boundary segregation in binary solutions suppressing both mentioned problems. This model enables the determination of grain boundary concentration and interfacial tension depending on grain boundary structure and temperature, using results of grain boundary simulations.

\section{Mixing Energies of Solute Atoms}

When one makes a calculation of grain boundary structure by simulation with semi-empirical potentials or by first principle methods, the energy of any atoms in the crystal is known, and the interface excess energy can be calculated very simply. To achieve this, we seek to model a bicrystal $\Omega$ of $\mathrm{Y}$ element atoms containing solute atoms of $\mathrm{X}$ element and grain boundary area $\mathrm{A}$. When pressure and volume

(1) Present adress : Centre de Recherche de Comptoir Lyon-Alemand-Louyot, 13 rue Montmorency, 75003 Paris, France 
variation during interface formation can be neglected, excess grain boundary energy $\mathrm{E}_{\mathrm{b}}$ can be written as follows :

$$
E_{b}\{\Omega\}=\frac{1}{A}\left[\sum_{\Omega} e^{Y_{i}}+\sum_{\Omega} e^{X_{i}}-\sum_{\Omega Y_{0}} e^{Y}-\sum_{\Omega X_{0}} e^{X}\right]
$$

where $\mathrm{e}_{\mathrm{i}}$ et $\mathrm{e}^{\mathrm{X}_{\mathrm{i}}}$ are respectively the energies of the atom number $\mathrm{i}$ of $\mathrm{Y}$ element and of the atom number $\mathrm{i}$ of $X$ element. $\Omega^{Y_{0}}$ and $\Omega^{X_{0}}$ are two equilibrium ensembles formed respectively by the same number of atoms $\mathrm{Y}$ and by the same number of atoms $\mathrm{X}$ than the bicrystal $\Omega$. $\Omega^{Y_{0}}$ and $\Omega \mathrm{Y}_{0}$ are in the same temperature and pression conditions as $\Omega$. $\mathrm{e}^{\mathrm{Y}}$ et $\mathrm{e}^{\mathrm{X}}$ are respectively the energies of any atom in $\Omega^{\mathrm{Y}_{0}}$ and in $\Omega^{X_{0}}$.

The computer simulations /1,2/ and High Resolution Electron Microscopy observations /3/ both, show that grain boundaries of a given type can be described in pure solids as a series of a small number of types of structural units, which frequency and degree of deformation vary with misorientation angle. (The multiplicity of structure manifestation /4/ can be seen as a lack or excess of one atom per unit structure. )

Computer simulation of solute solutions shows that, in the majority of cases, the types of structural units constructing grain boundaries in solid solutions are the same as in pure solids $/ 2,5 /$. The solute atoms substitute the solvent atoms or are located in interstitial sites. In order to characterize the susceptibility of a given atomic site to be occupied by a solute atom, it is convenient to determine the enthalpy of mixing associated to this type of site. To do this, we must first distinguish all the types of atomic sites in the bicrystal. We attribute the numbers $\mathrm{K}=2, \ldots, \mathrm{m}$ to the different atomic sites in the grain boundary and $\mathrm{K}=1$ to the sites in the bulk. Two atomic sites are of the same type if the energy of mixing (defined below) are equal in both sites. Let $N_{K}$ be the number of solute atoms in the sites of type $\mathrm{K}$ per unit area of grain boundary.

The mixing energy $\varepsilon_{\mathrm{K}}$ in this type of site can be defined as :

$$
\varepsilon_{\mathrm{K}}=\frac{\partial \mathrm{E}_{\mathrm{b}}}{\partial \mathrm{N}_{\mathrm{K}}}
$$

Mixing energy $\varepsilon_{\mathrm{K}}$ of an interstitial atom in the bicrystal $\Omega$ with a given composition can be calculated as :

$$
\varepsilon_{\mathrm{K}}\{\Omega\}=E_{\mathrm{b}}\{\Omega\}-E_{\mathrm{b}}\left\{\Omega^{\prime}\right\}-\mathrm{e}^{\mathrm{X}}
$$

where the atomic structure of $\Omega^{\prime}$ corresponds to $\Omega$ before one solute atom is mixed in the site of type K and $\mathrm{e}^{\mathrm{X}}$ has the same meaning as in equation (1).

In the case of substitutional segregation, $\varepsilon_{\mathrm{K}}$ can be written as :

$$
\varepsilon_{\mathrm{K}}\{\Omega\}=\mathrm{E}_{\mathrm{b}}\{\Omega\}+\mathrm{e}^{\mathrm{Y}}-\mathrm{E}_{\mathrm{b}}\left\{\Omega^{\prime}\right\}-\mathrm{e}^{\mathrm{X}}
$$

Knowing the values of $\varepsilon_{\mathrm{K}}$, we can write the variation of specific grain boundary free energy $F_{\mathrm{b}}$ with the number $\mathrm{N}_{\mathrm{K}}$ of solute atoms in different types of sites :

$$
\mathrm{F}_{\mathrm{b}}=\mathrm{E}^{\mathrm{Y}_{\mathrm{b}}}+\sum_{\mathrm{K}=1}^{\mathrm{m}} \mathrm{N}_{\mathrm{K}} \varepsilon_{\mathrm{K}}-\mathrm{TS}_{\mathrm{b}}
$$

where $\mathrm{E}_{\mathrm{b}}$ is the grain boundary energy in pure solvent and $\mathrm{S}_{\mathrm{b}}$ is the configurational grain boundary entropy (see § 3).

\section{Grain Boundary Site Occupation Ratio}

Let $Q_{1}$ be the number of sites for $X$ atoms in the bulk and $Q_{K}$, with $K=2, \ldots, m$ the number of sites of type $K$ per unit of grain boundary area. For simplicity let take grain boundary area $A$ equal to 1 . If we apply the statistical mechanics formulation corresponding to the grand canonical partition function for the solutes in our crystal, we obtain : 


$$
Z=\prod_{K=1}^{m} \frac{Q_{K} !}{N_{K} !\left(Q_{K}-N_{K}\right) !} \cdot \exp \left\{\frac{-E}{k T}\right\} \cdot \exp \left\{\frac{\mu}{k T} \sum_{K=1}^{m} N_{K}\right\} \cdot \exp \left\{\frac{\mu^{Y}}{k T} N^{Y}\right\}
$$

where $\mu^{X}$ and $\mu^{Y}$ are the chemical potentials for $X$ and $Y$ and:

$$
\mathrm{E}=\mathrm{N}^{\mathrm{X}} \mathrm{e}^{\mathrm{X}}+\mathrm{N}^{\mathrm{Y}} \mathrm{e}^{\mathrm{Y}}+\mathrm{E}^{\mathrm{Y}} \mathrm{b}+\sum_{\Omega} \mathrm{N}_{\mathrm{K}} \varepsilon_{\mathrm{K}}
$$

The partition function $\mathcal{Z}$ is, as usual, replaced by its maximal value, $\mathcal{Z}_{\mathrm{M}}$. Using Stirling formula we can write :

$$
\frac{\partial \operatorname{Ln} Z_{M}}{\partial N_{K}}=\ln \frac{Q_{K}-N_{K}}{N_{K}}+\frac{1}{k T} \cdot \frac{\partial}{\partial N_{K}}\left\{-E+\mu x \sum_{K=1}^{m} N_{K}+\mu^{Y} N^{Y}\right\}
$$

Taking into account that :

$$
\frac{\partial \mathrm{E}}{\partial \mathrm{N}_{\mathrm{K}}}=\varepsilon_{\mathrm{K}}+\mathrm{e}^{\mathrm{X}} \quad \text { and } \quad \mathrm{N}^{\mathrm{X}}=\sum_{\mathrm{K}=1}^{\mathrm{m}} \mathrm{N}_{\mathrm{K}}
$$

we obtain :

$$
\frac{\partial \ln Z_{M}}{\partial N_{K}}=\ln \frac{Q_{K}-N_{K}}{N_{K}}-\frac{1}{k T}\left[\varepsilon_{K}+e^{X}-\mu^{X}\right]
$$

Taking into account that at equilibrium $\frac{\partial \operatorname{Ln} \mathcal{Z}_{M}}{\partial N_{K}}=0$ for every $K$, we obtain a series of $m$ equations :

$$
\begin{aligned}
& \ln \frac{Q_{K}-N_{K}}{N_{K}}-\frac{1}{k T}\left[\varepsilon_{K}+e^{X}-\mu^{X}\right]=0 \quad \text { for } \quad K=1, \ldots ., m \\
& \text { or : } \quad \ln \frac{1-\mathrm{t}_{\mathrm{K}}}{\mathrm{t}_{\mathrm{K}}}-\frac{1}{\mathrm{kT}}\left[\varepsilon_{\mathrm{K}}+\mathrm{e}^{\mathrm{X}}-\mu^{\mathrm{X}}\right]=0 \quad \text { for } \mathrm{K}=1, \ldots, \mathrm{m}
\end{aligned}
$$

where $t_{K}=\frac{N_{K}}{Q_{K}}$ is the $K$. site occupation ratio. This series can be rewritten for $K=2, \ldots, m$ as :

$$
\ln \frac{1-\mathrm{t}_{\mathrm{K}}}{\mathrm{t}_{\mathrm{K}}}-\frac{1}{\mathrm{kT}}\left[\varepsilon_{\mathrm{K}}+\mathrm{e}^{\mathrm{X}}-\mu^{\mathrm{X}}\right]=\ln \frac{1-\mathrm{t}_{1}}{\mathrm{t}_{1}}-\frac{1}{\mathrm{kT}}\left[\varepsilon_{1}+\mathrm{e}^{\mathrm{X}}-\mu^{\mathrm{X}}\right] .
$$

Rearranging the terms, we can calculate the $\mathrm{K}$ type site occupation ratio as a function of temperature and bulk concentration $t_{1}$ as :

$$
\mathrm{t}_{\mathrm{K}}=\frac{\mathrm{t}_{1} \cdot \exp \left[\frac{-\varepsilon_{\mathrm{SK}}}{\mathrm{kT}}\right]}{1-\mathrm{t}_{1}+\mathrm{t}_{1} \cdot \exp \left[\frac{-\varepsilon_{S K}}{\mathrm{kT}}\right]} \text {, where } \varepsilon_{\mathrm{SK}}=\varepsilon_{\mathrm{K}}-\varepsilon_{1} \text {. }
$$

The superficial density of solute in the grain boundary can be calculated as:

$$
\mathrm{R}=\sum_{\mathrm{K}=2}^{\mathrm{m}} \mathrm{Q}_{\mathrm{K}} \mathrm{t}_{\mathrm{K}}
$$

If one defines grain boundary concentration $C_{b}$ as :

$$
\mathrm{C}_{\mathrm{b}}=\frac{\mathrm{R}}{\mathrm{P}}
$$


where $P$ is a mean surface atomic density of the solvent $Y$ in a crystal, grain boundary solute concentration can be expressed as :

$$
C_{b}=\frac{1}{P} \sum_{K=2}^{m} Q_{K} \frac{t_{1} \cdot \exp \left[\frac{-\varepsilon_{S K}}{k T}\right]}{1-t_{1}+t_{1} \cdot \exp \left[\frac{-\varepsilon_{S K}}{k T}\right]}
$$

This equation is similar to the well known McLean equation $/ 6 /$, but it enables to take into account the differences between the energy of segregation in the different types of segregation sites. The major difficulty in using this result is that it is necessary to know the bulk concentration for grain boundary concentration determination.

In cases the chemical potential of solute is imposed, grain boundary concentration can be calculated by a simpler method than given by equation (17). By rearranging (13) one can write :

$$
\mathrm{t}_{\mathrm{K}}=\frac{1}{1+\exp \left[\frac{\varepsilon_{\mathrm{K}}+\mathrm{e}^{\mathrm{X}}-\mu^{\mathrm{X}}}{\mathrm{kT}}\right]}
$$

and by taking into account (16):

$$
C_{b}=\frac{1}{P} \sum_{K=2}^{m} Q_{K} \frac{1}{1+\exp \left[\frac{\varepsilon_{K}+e^{X}-\mu^{X}}{k T}\right]}
$$

When the chemical potential is fixed by establishing an equilibrium between the bicrystal of solute solution $\mathrm{X}-\mathrm{Y}$ and pure $\mathrm{X}$ element, $\mu^{\mathrm{X}}$ is equal to $\mathrm{e}^{\mathrm{X}}$ and we obtain :

$$
C_{b}=\frac{1}{P} \sum_{K=2}^{m} Q_{K} \frac{1}{1+\exp \left[\frac{\varepsilon_{K}}{k T}\right]}
$$

The advantage in using equation (20) is that we can calculate the grain boundary concentration $\mathrm{C}_{b}$ without knowing the bulk solute concentration $\mathrm{t}_{1}$.

\section{Bicrystal Entropy}

The configurational entropy $S_{c}$ of bicrystal can be calculated as follows :

$$
\mathrm{S}_{\mathrm{c}}=\mathrm{k} \prod_{\mathrm{K}=1}^{\mathrm{m}} \ln \frac{\mathrm{Q}_{\mathrm{K}} !}{\left(\mathrm{Q}_{\mathrm{K}}-\mathrm{N}_{\mathrm{K}}\right) !}
$$

and using the Stirling formula we obtain:

$$
\mathrm{S}_{\mathrm{c}}=-\mathrm{k} \sum_{\mathrm{K}=1}^{\mathrm{m}} \mathrm{Q}_{\mathrm{K}}\left[\ln \left(1-\mathrm{t}_{\mathrm{K}}\right)+\mathrm{t}_{\mathrm{K}} \cdot \ln \frac{\mathrm{t}_{\mathrm{K}}}{1-\mathrm{t}_{\mathrm{K}}}\right]
$$

Total entropy of bicrystal S can be written as :

$$
\mathrm{S}=\mathrm{S}_{\mathrm{c}}+\overline{\mathrm{S}}
$$

where $\overline{\mathrm{S}}$ is the excess entropy of bicrystal which can be obtained from theoretical models or from molecular dynamics simulations. In order to take $\overline{\mathrm{S}}$ into account, it is convenient to calculate the excess entropy per solute atom in a site of type $\mathrm{K}$ : 


$$
\overline{\mathrm{s}}_{\mathrm{K}}=\frac{\partial \overline{\mathrm{s}}}{\partial \mathrm{N}_{\mathrm{K}}}
$$

Using (23) one can write :

$$
\overline{\mathrm{S}}=\mathrm{S}_{\mathrm{b}}+\sum_{\mathrm{K}=1}^{\mathrm{m}} \overline{\mathrm{s}}_{\mathrm{K}}
$$

where $S \mathrm{Y}_{b}$ is the grain boundary entropy of a bicrystal of pure solvent. Substituting (25) in equation (5), we obtain:

$$
F_{b}=E^{Y_{b}}+\sum_{K=1}^{m} N_{K} f_{K}-T\left(S_{c}+S Y_{b}\right)
$$

where $f_{K}=\varepsilon_{K}-T \bar{s}_{K}$ is the excess free energy of mixing in a site of type $K$. Finally, using (25), we can rewrite equations (17) and (20) as :

$$
\begin{aligned}
& C_{b}=\frac{1}{P} \sum_{K=2}^{m} Q_{K} \frac{1}{1+\exp \left[\frac{f_{K}-\mu x}{k T}\right]} \\
& C_{b}=\frac{1}{P} \sum_{K=2}^{m} Q_{K} \frac{t_{1} \cdot \exp \left[\frac{-f_{S K}}{k T}\right]}{1-t_{1}+t_{1} \cdot \exp \left[\frac{-f_{S K}}{k T}\right]}
\end{aligned}
$$

where $f_{S K}=f_{K}-f_{1}$ is excess free energy of segregation in site of type $K$.

\section{Interfacial Tension}

Interfacial tension $\sigma$ can be calculated, using its definition, as :

$$
\sigma=\frac{\partial \mathrm{F}}{\partial \mathrm{A}}
$$

where $\mathrm{F}$ is the Helmholtz free energy of a bicrystal calculated as a function of grain boundary area at constant number of atoms $\mathrm{N}^{\mathrm{Y}}$ and $\mathrm{NX}^{\mathrm{X}}$.

In this case, the number of solute atoms in the bulk $\mathrm{N}_{1}$ can be calculated as :

$$
N_{1}=N^{X}-A \sum_{K=2}^{m} N_{K}
$$

and the free energy $F$ of bicrystal $\Omega$ can be written as:

$$
\mathrm{F}=\mathrm{NX}^{\mathrm{X}} \mathrm{e}^{\mathrm{X}}+\dot{\mathrm{N}^{\mathrm{Y}}} \mathrm{e}^{\mathrm{Y}}+\left[\mathrm{NX}-\mathrm{A} \sum_{\mathrm{K}=2}^{\mathrm{m}} \mathrm{N}_{\mathrm{K}}\right] \cdot \varepsilon_{1}-\mathrm{TS}_{1}+\mathrm{A} \mathrm{F}_{\mathrm{b}}
$$

where $S_{1}$ is the grain interior entropy. Replacing (28) and (5) into (26), we can calculate interfacial tension as :

$$
\sigma=E^{Y_{b}}+\sum_{K=2}^{m} N_{K} \varepsilon_{S K}-T_{S_{b}}
$$

where $\varepsilon_{S K}=\varepsilon_{K}-\varepsilon_{1}$ is usually called energy of segregation. 
Equation (29) is a very useful one, because it enables a calculation of interfacial tension to be made when the energies of mixing $\varepsilon_{K}$ and the numbers $N_{K}$ of solute atoms per area unit in every type of sites are known. The values of $\varepsilon_{K}$ can be determined by using equation (4) and the method of calculation of $N_{K}$ for different temperatures is presented otherwise /1/.

\section{Conclusion}

In this paper we have proposed a new formulation of grain boundary thermodynamical description applicable to systems exhibiting various types of segregation sites. This description consists in gathing together the energies of some atoms in the ensembles corresponding to simple physical notions such as grain boundary energy $\left(E_{b} Y\right)$ or energy of mixing $\left(\varepsilon_{K}\right)$ in grain boundary sites of different types. By this method, we can make thermodynamic calculations such as the determination of grain boundary tension and composition according to temperature and grain boundary structure. When one substitutes our grain boundary description for that used in the classical models, the proposed equations become equivalent to classic results :

- equation (17) reduces to McLean equation $/ 6 /$;

- formula (29) becomes Gibbs formula $\Pi /$.

Using equation (17), we can compare directly the results of calculated values of energy of mixing in different types of sites $\varepsilon_{K}$ and the calculated superficial density of given types of sites $Q_{K}$ to experimental grain boundary concentration measurements corresponding to different temperatures and bicrystal misorientations /1/. Formula (29) can be used for comparison of calculated values of $\varepsilon_{\mathrm{K}}$ and $\mathrm{Q}_{\mathrm{K}}$ with the variation of interfacial tension measured for different grain boundary concentrations.

\section{REFERENCES}

1/ Nowicki T., Biscondi M., J. Physique, this issue.

12/ Sutton A.-P., Vitek V., Phil. Trans. R. Soc. Lond. A309 (1983) 1.

13/ Penisson J.-M., Nowicki T., Biscondi M., Phil. Mag. 58 (1988) 947.

14/ Wang G.-J., Sutton A.-P., Vitek V., Acta Metall. 32 (1984) 1093.

/5/ Sutton A.-P., Vitek V., Acta Metall. $\underline{30}$ (1982) 2011.

16/ McLean D., "Grain Boundaries in Metals" (Clarendon Press, Oxford, 1957) p.118.

n/ Gibbs J.-W., "Collected Works ", Vol. I (Longmans, 1931). 